Metal nanoparticle mediated space charge and its optical control in an organic holeonly device

G. Ligorio, M. V. Nardi, R. Steyrleuthner, D. Ihiawakrim, N. Crespo-Monteiro', M. Brinkmann, D. Neher, and N. Koch'

Citation: Appl. Phys. Lett. 108, 153302 (2016); doi: 10.1063/1.4945710

View online: http://dx.doi.org/10.1063/1.4945710

View Table of Contents: http://aip.scitation.org/toc/apl/108/15

Published by the American Institute of Physics 


\title{
Metal nanoparticle mediated space charge and its optical control in an organic hole-only device
}

\author{
G. Ligorio, ${ }^{1}$ M. V. Nardi, ${ }^{1}$ R. Steyrleuthner, ${ }^{2}$ D. Ihiawakrim, ${ }^{3}$ N. Crespo-Monteiro, ${ }^{4, a)}$ \\ M. Brinkmann, ${ }^{4}$ D. Neher, ${ }^{2}$ and N. Koch ${ }^{1,5, b)}$ \\ ${ }^{1}$ Institut für Physik \& IRIS Adlershof, Humboldt-Universität zu Berlin, Brook-Taylor Str. 6, 12489 Berlin, \\ Germany \\ ${ }^{2}$ Institute of Physics and Astronomy, Universität Potsdam, Karl-Liebknecht Str. 24, 14476 Potsdam, Germany \\ ${ }^{3}$ Institut de Physique et de Chimie des Matériaux de Strasbourg, Université de Strasbourg, CNRS UMR 7504, \\ 23 rue du Loess, BP 43, 67034 Strasbourg, Cedex2, France \\ ${ }^{4}$ Institut Charles Sadron CNRS, 23 rue du Loess, 67034 Strasbourg, France \\ ${ }^{5}$ Helmholtz-Zentrum Berlin für Materialien und Energie GmbH, Erneuerbare Energien, \\ Albert-Einstein Str. 15, 12489 Berlin, Germany
}

(Received 31 January 2016; accepted 28 March 2016; published online 11 April 2016)

\begin{abstract}
We reveal the role of localized space charges in hole-only devices based on an organic semiconductor with embedded metal nanoparticles (MNPs). MNPs act as deep traps for holes and reduce the current density compared to a device without MNPs by a factor of $10^{4}$ due to the build-up of localized space charge. Dynamic MNPs charged neutrality can be realized during operation by electron transfer from excitons created in the organic matrix, enabling light sensing independent of device bias. In contrast to the previous speculations, electrical bistability in such devices was not observed. C 2016 AIP Publishing LLC. [http://dx.doi.org/10.1063/1.4945710]
\end{abstract}

One type of next-generation non-volatile memory elements, governed by resistive bistability, has been suggested to comprise an organic semiconductor (small molecules or polymers) layer with embedded metal nanoparticles (MNPs), sandwiched between two electrodes. ${ }^{1-5}$ The writing, reading, and erasing of information is then based on resistive bistability of the element. The use of organic materials offers the advantages of low fabrication costs, easy scalability, and printability on flexible supports. ${ }^{6,7}$ Moreover, the simple twoterminal structure allows the realization of a simple cross-bar architecture for memory arrays. ${ }^{8,9}$

Significant effort was devoted to reveal the resistive switching mechanism in these bistable devices, and two main-mutually exclusive-models were put forward. One mechanism is based on space-charge build-up within the device during operation. The switching between the high- and low-resistance state is then related to bias-dependent charging/de-charging of the embedded MNPs. ${ }^{10,11}$ The second suggested mechanism is based on the filament formation/rupture. ${ }^{12,13}$ The electrical switching to the low-resistance state is explained by the formation of a highly conductive localized path (filament). The filament formation is driven by electrochemical dissolution of active electrodes (e.g., $\mathrm{Al}, \mathrm{Cu}$, and $\mathrm{Ag}$ ) into the organic matrix. ${ }^{14,15}$

Noteworthy, both mechanisms were alternatingly used to explain the function of devices that exhibited almost identical electrical characteristics. Support for the second model came recently from the work by Nau et al., who studied the switching behavior of prototypical devices based on polymer and small molecule matrices with and without embedded

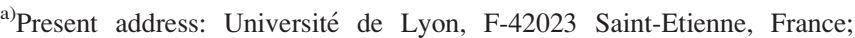
CNRS, UMR 5516, Laboratoire Hubert Curien, 18 rue Pr. Lauras F-42000 Saint-Etienne, France; and Université de Saint-Etienne, Jean-Monnet, F42000 Saint-Etienne, France.

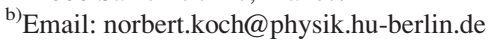

MNPs. ${ }^{16}$ Independently of the presence of MNPs, the devices displayed the same electrical characteristics, reminiscent of this type of non-volatile memory elements as also reported by many other groups. With impedance spectroscopy studies, they could unequivocally demonstrate that the resistive switching is due to the formation/rupture of filaments formed from electrode material, the only prerequisite being the proper electrical pre-conditioning of the device.

On the other hand, the proposed switching mechanism based on charging/de-charging of the MNPs lacks solid evidence. This, in part, is due to the poor structural/morphological characterization of the device structures employed so far. In a previous study, we applied ultraviolet photoemission spectroscopy on samples with high morphological precision, where MNPs were deposited onto an organic semiconductor. These studies indeed revealed space charge due to the accumulation of holes on the MNPs. ${ }^{17}$ Furthermore, it was demonstrated that charge neutrality of the MNPs can be reestablished by exciton-mediated electron transfer from the organic semiconductor to the MNPs upon illumination with light absorbed by the organic semiconductor.

Here, we report the electrical characteristics of reliably fabricated organic matrix/MNP two-terminal devices with defined MNP location, size, and density. The choice of the materials (which does not provide ion dissolution) and the performed electrical characterization were such that filament formation was excluded. We confirm that, indeed, space charge is accumulated on MNPs during device operation but that the devices lack electrical bistability. Nonetheless, the removal of the space charge by illumination, and the corresponding resistance reduction, is also evidenced, opening alternative routes for the control of electrical conductivity with light.

Hole-only devices were prepared on glass substrates with a patterned indium-tin-oxide (ITO) layer. The ITO/glass 
substrates were cleaned by sequential sonication in acetone, detergent, deionized water, and isopropyl alcohol before subsequent oxygen plasma treatment ( $200 \mathrm{~W}$ for $3 \mathrm{~min}$ ). ITO was coated with $\sim 50 \mathrm{~nm}$ thick layer of poly(3,4-ethylenedioxythiophene)/poly(styrene sulfonate) PEDOT:PSS (Clevios AI 4083) by spin-casting ( $30 \mathrm{~s}$ at $1500 \mathrm{rpm}$ ). The substrates were first transferred to a nitrogen filled glove box and annealed at $180^{\circ} \mathrm{C}$ for $10 \mathrm{~min}$, and then-without air exposure-introduced in a high vacuum (base pressure $1 \times 10^{-6} \mathrm{mbar}$ ) preparation chamber.

The preparation of reference devices (without MNP) progressed in three steps: (i) vacuum deposition of $120 \mathrm{~nm}$ of the hole transport organic semiconductor 4,4-bis[N-(1-naphthyl)-N-phenyl-amino]diphenyl ( $\alpha$-NPD, Sigma Aldrich), thermal evaporation of (ii) $10 \mathrm{~nm} \mathrm{MoO}_{3}$ and (iii) $100 \mathrm{~nm}$ gold through a shadow. A schematic illustration of the device structure is depicted in Figure 1(a). The device-pixel area was $0.15 \mathrm{~cm}^{2}$ (10 pixels produced on each substrate) as defined by the geometric overlap between the bottom ITO strips and the metal top electrodes. The whole process was carried out without breaking the vacuum; deposition rates were controlled by the respective evaporation source temperature and monitored with quartz crystal microbalances.

Devices with embedded gold nanoparticles (AuNPs) were fabricated in parallel with reference devices, which were kept masked during AuNPs deposition. The electrodes (bottom and top) of both the device types are therefore identical. The device structure is depicted in Figure 1(b). The defined AuNPs were formed by the deposition of a small amount of Au by thermal evaporation on top of a $60 \mathrm{~nm}$ thick $\alpha$-NPD layer. Subsequently, $60 \mathrm{~nm}$ of $\alpha$-NPD and the top electrode were deposited. The formation of AuNPs with defined location within the devices was confirmed by transmission electron microscopy (TEM). Figure 1(d) shows a top view of a TEM bright field image of an equivalent sample prepared on a TEM copper-grid. Consistently with our previous investigations, ${ }^{17}$ we find that Au does not percolate into the organic layer, i.e., the non-perculated AuNPs form a

(a)

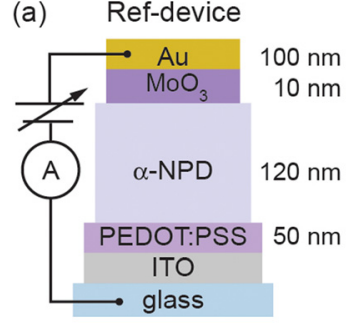

(b) AuNPs-device

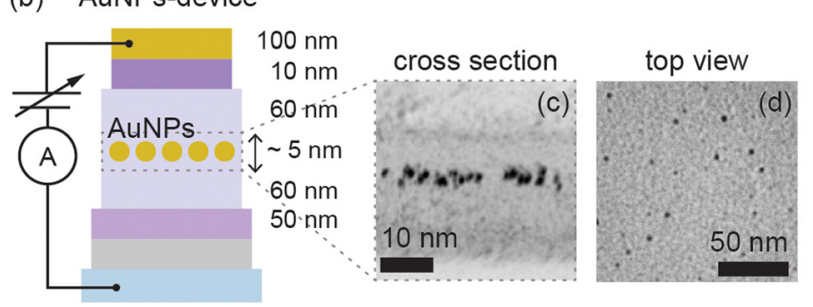

FIG. 1. Top view (a) and cross sectional (b) typical TEM cross-sectional slice extracted from the 3D reconstruction obtained by the electron tomography for AuNPs within $\alpha$-NPD. The schematic of the experimental setup is illustrated in (c). UV-VIS absorbance spectra of $\alpha$-NPD in (d). sharp layer within the amorphous $\alpha$-NPD layer, as illustrated by the cross sectional TEM tomography projection shown in Figure 1(c). Statistical analysis gives an average AuNP diameter $\left(d_{\mathrm{AuNP}}\right)$ of $(1.0 \pm 0.1) \mathrm{nm}$. The devices with nanoparticles, therefore, comprise a horizontal structure with an $\alpha$ NPD length $L=120 \mathrm{~nm}$ and a narrow $(h=5 \mathrm{~nm})$ interlayer of dispersed AuNPs positioned at $L / 2$ [see Figure 1(b)].

After sample preparation, the current density vs. voltage $(j \mathrm{U})$ characteristics of the reference devices and the AuNPsdevices were measured by a computer controlled Keithley 2400 sourcemeter, in a nitrogen filled glove box, without exposing the sample to air at any stage of the experiment. The $j \mathrm{U}$ curves are measured with one sweep in stepped bias mode. The $j \mathrm{U}$-measurements were carried out at room temperature under three different conditions: (i) dark, (ii) illuminating the device through the glass with a red laser (wavelength $\lambda=685 \mathrm{~nm}$ ), and (iii) a blue laser $(\lambda=405 \mathrm{~nm}$ ). The laser beams were broadened with an optical lens in order to irradiate the whole active device-pixel; the light power density was $\sim 0.05 \mathrm{~mW} \mathrm{~cm} \mathrm{~cm}^{-2}$. Note that the power density was sufficient to reach the saturation of the observed effect (vide infra). The wavelengths of the light were chosen according to the optical properties of the organic material, in order to be within (blue) and outside (red) the $\alpha$-NPD optical absorption. Figure 1(e) shows the ultraviolet-visible (UVVIS) absorbance spectrum of a $60 \mathrm{~nm}$ thick $\alpha$-NPD film and the vertical lines indicate the employed light wavelengths.

The $j \mathrm{U}$ characteristics of the reference device (topmost curves with filled symbols) are shown on a semi-log scale in Figure 2(a) and a log-log scale in Figure 2(b). The arrows in Figure 2(a) indicate the applied bias sweep. No difference is observed in the $j \mathrm{U}$ curves with respect to the sweep direction. Forward bias indicates the bottom electrode (ITO/ PEDOT:PSS) to be negatively biased, i.e., positive charges injected from the top electrode $\left(\mathrm{MoO}_{3} / \mathrm{Au}\right)$, as schematically shown in Figure 2(c). The $j \mathrm{U}$ curve is asymmetric due to the different top and bottom electrodes. While $\alpha$-NPD is Fermilevel pinned at both the electrodes, the hole injection from $\mathrm{MoO}_{3} / \mathrm{Au}$ is yet to be improved over that from PEDOT:PSS due to the doping of the organic semiconductor close to the interface. ${ }^{18,19}$

When charge carriers are injected through ohmic contacts, the current density is expected to be limited by the accumulation of injected charge carriers in the bulk. In an ideal trap-free medium, this is described by the Mott-Gurney law

$$
j_{\mathrm{MG}}=\frac{9}{8} \varepsilon_{0} \varepsilon_{\mathrm{r}} \mu \frac{\mathrm{U}^{2}}{L^{3}},
$$

where $\varepsilon_{0}$ is the vacuum permittivity, $\varepsilon_{\mathrm{r}}$ is the dielectric constant of the semiconductor, $\mu$ its carrier mobility, and $L$ is the distance between the electrodes. Consistently, the reference devices' $j \mathrm{U}$-curves at forward bias match this law well [see the log-log plot in Figure 2(b)]. The mobility $\mu_{\alpha-\mathrm{NPD}}$ of the pristine organic layer is extracted from the best fit, assuming $L=120 \mathrm{~nm}$ and $\varepsilon_{r}=3$ (Ref. 20) in good agreement with literature as $7.5 \times 10^{-6} \mathrm{~cm}^{2} \mathrm{~V}^{-1} \mathrm{~s}^{-1}$. $20-22$

Reference devices do not exhibit any resistance bistability (the resistance does not abruptly change upon a critical 

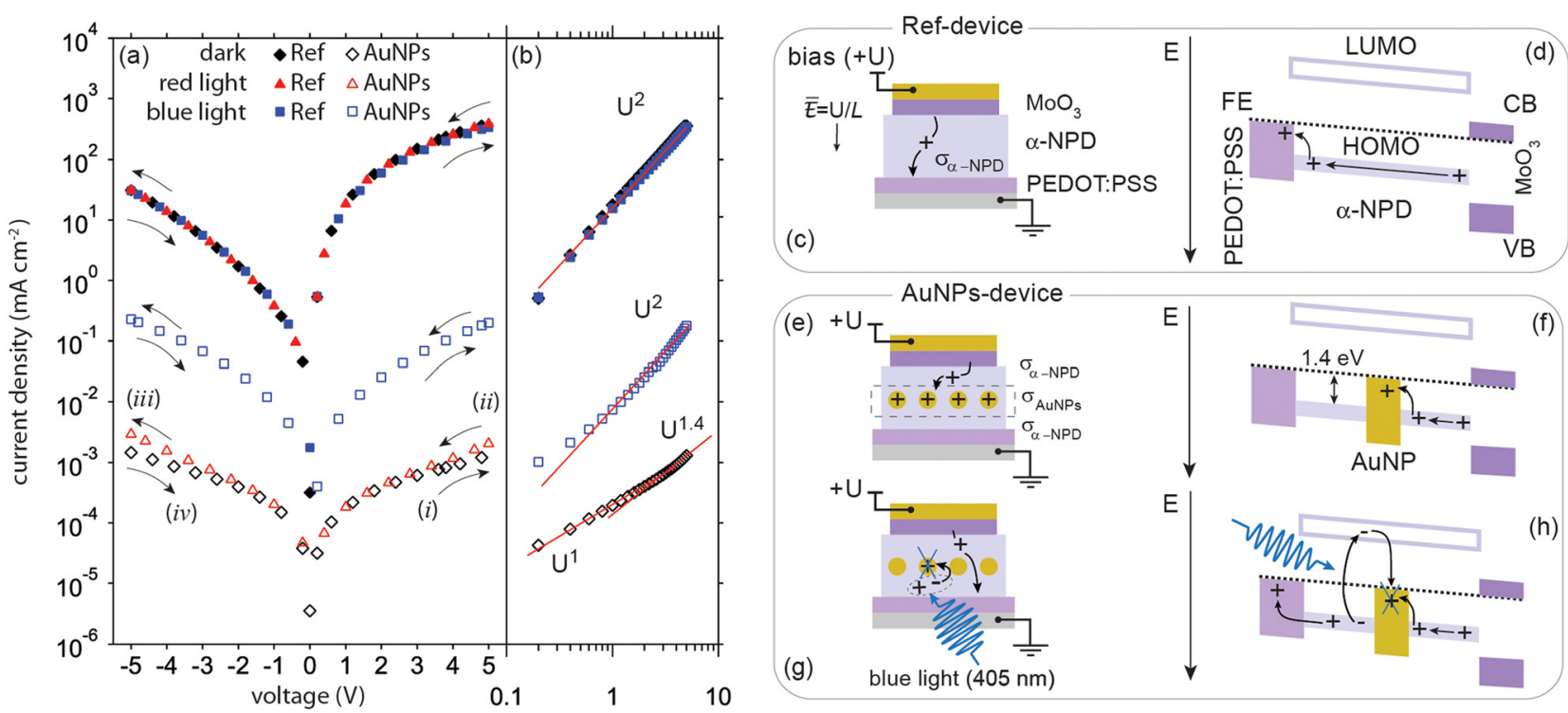

(d)
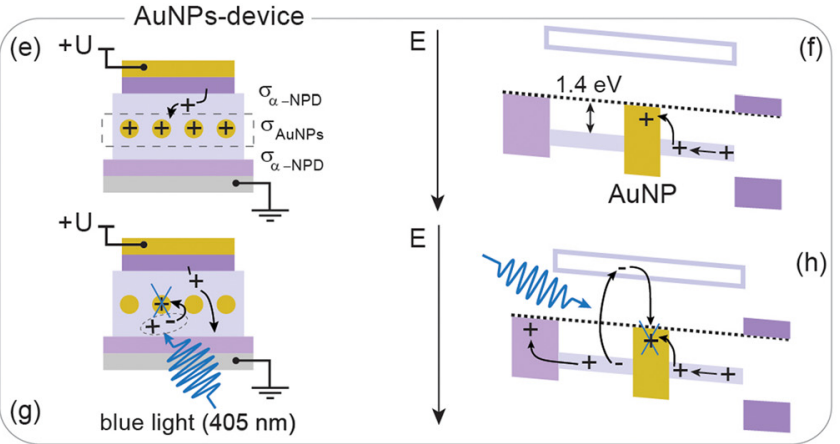

FIG. 2. The $j \mathrm{U}$ characteristic of the reference device and the AuNPs-device on a semi-log and a log-log scale, respectively, in (a) and (b). The $j \mathrm{U}$ curves were measured in dark as well as with illumination (maximum light power available, close to saturation). The schematic for the reference upon forward bias and its energy diagram in (c) and (d), respectively. The positive carriers ("+" symbol) are injected from the top electrode. The AuNPs-device schematic and its energy level diagram in dark are depicted in (e) and (f), respectively. The hole-injection barrier from AuNPs to $\alpha$-NPD is indicated in (f). The case relative to the AuNPsdevice upon blue light illumination in (g) and (h). The promotion of electrons ("-" symbol) in the LUMO of $\alpha$-NPD via light absorption is shown in (h).

value of bias), ${ }^{23}$ independent of bias sequence and condition. In addition, neither red nor blue light irradiation affects the $j \mathrm{U}$ curves compared to the dark, i.e., the photocurrent is negligible.

We now turn towards the device with the AuNPs interlayer, see Figure 1(b). The bottommost curves in Figure 2(a) are the $j \mathrm{U}$ curves of the AuNPs-device measured in dark (empty black diamond symbols) and with illumination with red (empty red triangles) and blue light (empty blue squares). As for the reference devices, no resistance bistability is observed ${ }^{23}$ instead, the overall current density of the AuNPsdevice is ca. four orders of magnitude lower in the dark and with red light illumination.

The differences in the $j \mathrm{U}$ curves of the reference and the AuNPs-devices [represented in Figures 2(c) and 2(e), respectively] are the result of the AuNPs incorporation within the $\alpha$-NPD. AuNPs behave as deep-traps for the charge carriers (holes) because of the energy alignment between $\mathrm{Au}$ and $\alpha$ NPD, ${ }^{24}$ see Figure 2(f). The Fermi level of the AuNPs lies between the molecular frontier energy levels. For holes, it is therefore energetically favorable to become trapped and remain on the AuNPs, as the hole-injection barrier from AuNPs into $\alpha$-NPD is $1.4 \mathrm{eV}{ }^{25}$ Therefore, a localized space charge formed by positively charged AuNPs occurs in the middle of the organic layer, thereby inhibiting the drift of holes through the device. Furthermore, the thermal deposition of AuNPs onto the organic layer changes its electronic properties. The interaction between AuNPs and the $\alpha$-NPD results in an increased width of the conduction level energy distribution, described by the effective width parameter $\sigma,{ }^{17}$ which reduces carrier mobility. Therefore, the interlayer comprising AuNPs with neighboring $\alpha$-NPD has a wider $\sigma$ with respect to the $\sigma_{\alpha \text {-NPD }}$ of the pristine $\alpha$-NPD. For convenience, we indicate as $\sigma_{\text {AuNPs }}$ the effective parameter of the midway-localized AuNPs $/ \alpha$-NPD interlayer of at least $5 \mathrm{~nm}$ thickness (with $\sigma_{\mathrm{AuNPs}}>\sigma_{\alpha \text {-NPD }}$ ). The overall carrier mobility depends on $\sigma$, as well as temperature $T$, carrier density $p$, and the electric field $E$, i.e., $\mu=\mu(T, p, E, \sigma){ }^{26,27}$ While the space charge on the AuNPs globally reduces $j$ through the device due to an electric field re-distribution, the carrier mobility decreases locally at and near the interlayer with respect to the mobility of the pristine molecular bulk. Furthermore, as direct consequence of the carrier-trapping nature of the AuNPs, $j$ can no longer be described by the simple Mott-Gurney law, see Figure 2(b) and Ref. 23.

Red light illumination has no notable impact on the $j \mathrm{U}$ characteristics, see Figure 2(a). When AuNPs-devices are measured with blue light illumination (absorbed by $\alpha$-NPD), the current density instantly increases by a factor of $\sim 100$ with respect to one measured in dark, and immediately returns to the lower value when the light is switched off. The higher current measured upon illumination cannot be attributed to the photogeneration at the electrodes interface, because no effect was seen for the reference devices. Instead, we ascribe the observed effect to the photogeneration and dissociation of excitons created in $\alpha$-NPD. Here, excitons generated on $\alpha$ NPD diffuse to the positively charged MNP layer, where they

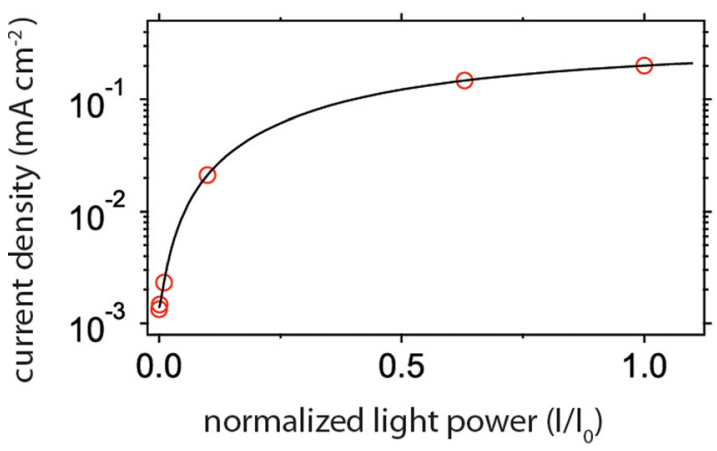

FIG. 3. AuNPs-device current density (at $5 \mathrm{~V}$ bias) $v s$. incident normalized light power (blue light). The solid line is a guide for the eyes. 
dissociate by donating an electron to an MNP as shown in the schematic Figure 2(h); this removes positive charge trapped on the AuNPs while at the same time creating a mobile hole on the $\alpha$-NPD matrix. Figure 3 shows in a semi-log plot the current density measured at $5 \mathrm{~V}$ versus the light intensity. Light intensity was gradually lowered via gray filters. The solid line serves as a guide for the eye. The maximum light intensity available in our experiments is close to reach the saturation regime, i.e., establishing almost charge-neutral AuNPs. Since now essentially all charge traps are dynamically removed, the $j \mathrm{U}$ curve can again be well described by the Mott-Gurney law, see Figure 2(b) and Ref. 23. Even under illumination with blue light of (close-to) saturation power, $j$ is still about 3 orders of magnitude lower compared to the reference device. This is due to the increased width of the conduction level energy distribution $\left(\sigma_{\mathrm{AuNPs}}\right)$ in proximity of the MNP layer, due to the AuNP-organic interaction as discussed above.

Despite the above-demonstrated ability to (optically-) remove the space charge localized at the AuNPs interlayer, the devices do not display any bistability. It is worth to notice that the materials used for electrodes (ITO/PEDOT:PSS and $\mathrm{MoO}_{3} / \mathrm{Au}$ ) do not allow for electrochemical dissolution of ions, which leads to the filament formation. This suggests that the MNPs charging/discharging mechanism is not a sufficient condition for resistivity switching.

In order to prove whether filament-formation is a necessary condition for bistability, a modified device was fabricated: the bottom contact (ITO/PEDOT:PSS) remained the same as

(a)
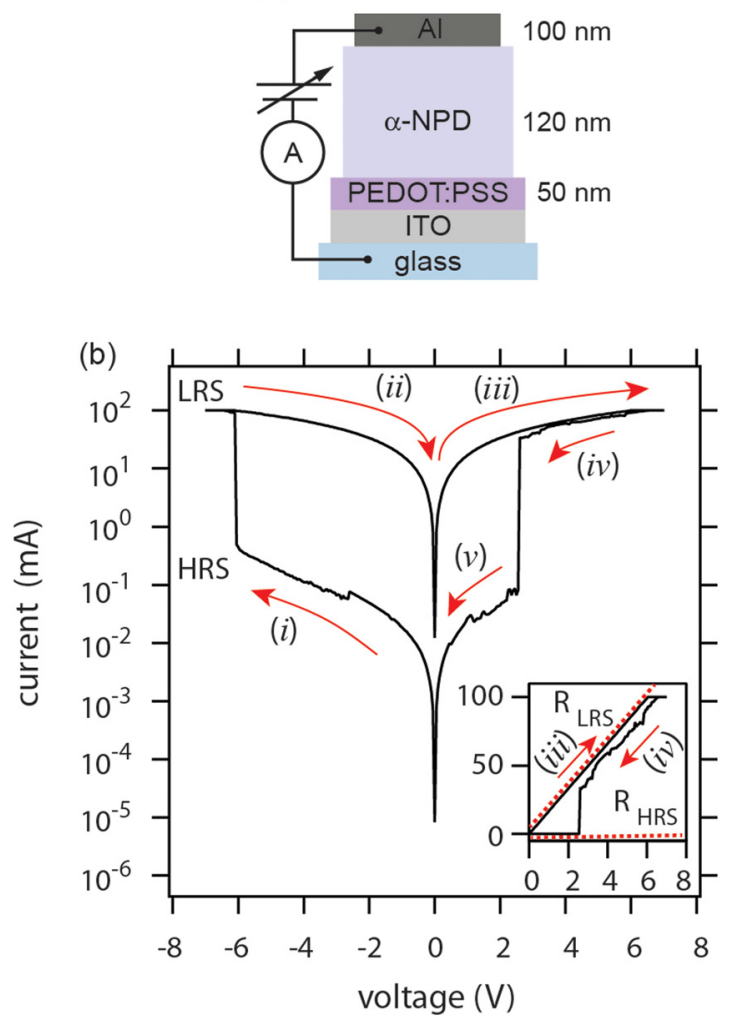

FIG. 4. Schematic of the modified device with Al top-electrode in (a). The device's $I U$ curve on a semi-log scale after electrical conditioning plotted in (b). The indexed arrows indicate the bias sweep. The inset in (b) plots the $i U$ curve at forward bias in linear scale. The lines are linear fit. in the devices described above, but the top contact was substituted with an electrochemical active material, $\mathrm{Al}$ (thickness $100 \mathrm{~nm}){ }^{28,29}$ The layer of $\alpha$-NPD had the same nominal thickness $(120 \mathrm{~nm})$ as the previous devices. No metal NPs were intentionally introduced within the $\alpha$-NPD film. The device's structure is illustrated in Figure 4(a). Figure 4(b) displays the $j \mathrm{U}$ curve of the ITO/PEDOT:PSS/ $\alpha-\mathrm{NPD} / \mathrm{Al}$ after adequate conditioning (by means of repeated set/reset cycles, see supplementary material). In contrast to the above-discussed devices with electrochemically inert electrodes (PEDOT:PSS and $\mathrm{MoO}_{3}$ ), the device with Al-electrode displays resistive bistability. The insert in Figure 4(b) displays the measured current in linear scale upon forward bias. The resistance values for the high-resistance state (HRS) and the low-resistance state (LRS) are calculated from the curve linear best-fit (dashed lines); $R_{\mathrm{LRS}} \sim 2.8 \times 10^{5} \Omega$ and $R_{\mathrm{HRS}} \sim 4.9 \times 10^{2} \Omega$. Sebastian et al. reported on a conceptually identical device $(\mathrm{ITO} / \alpha-\mathrm{NPD} / \mathrm{Al}) .{ }^{29}$ Accordantly, the impedance spectroscopy analysis reveals the filamentary nature of the switching.

In summary, we realized hole-only devices based on $\alpha$ NPD with spatially defined AuNPs to unravel whether charge accumulation on the metal nanoparticles can be used to realize resistively switching non-volatile memory elements. We find that AuNPs do behave as deep traps for holes and that a space charge within the device can be built up. However, electrical bistability is not observed, confirming that the resistance switching mechanism in such type of devices is due to filament formation. Nonetheless, the space charge on the AuNPs can be reduced (and eventually removed) by electron transfer from excitons created in the organic layer by light absorption. The dynamic range of wavelength-dependent light-controlled current in these very simple and easy-to-make two-terminal devices covers two orders of magnitude at any applied voltage and polarity. By further optimization of organic semiconductor materials and metal nanoparticle size and distribution, highly sensitive light sensors should become possible.

The authors gratefully acknowledge the financial support from the European Commission FP7 Project HYMEC (Grant No. 263073), the Sfb951 (DFG), and the Helmholtz Energy Alliance "Hybrid Photovoltaics."

${ }^{1}$ L. P. Ma, J. Liu, and Y. Yang, Appl. Phys. Lett. 80, 2997 (2002).

${ }^{2}$ J. Ouyang, C.-W. Chu, C. R. Szmanda, L. Ma, and Y. Yang, Nat. Mater. 3, 918 (2004).

${ }^{3}$ J. C. C. Scott and L. D. Bozano, Adv. Mater. 19, 1452 (2007).

${ }^{4}$ Q.-D. Ling, D.-J. Liaw, C. Zhu, D. S.-H. Chan, E.-T. Kang, and K.-G. Neoh, Prog. Polym. Sci. 33, 917 (2008).

${ }^{5}$ P. Heremans, G. H. Gelinck, R. Müller, K.-J. Baeg, D.-Y. Kim, and Y.-Y. Noh, Chem. Mater. 23, 341 (2011).

${ }^{6}$ M. Kang, K.-J. Baeg, D. Khim, Y.-Y. Noh, and D.-Y. Kim, Adv. Funct. Mater. 23, 3503 (2013).

${ }^{7}$ Y. Ji, D. F. Zeigler, D. S. Lee, H. Choi, A. K.-Y. Jen, H. C. Ko, and T.-W. Kim, Nat. Commun. 4, 2707 (2013).

${ }^{8}$ T.-W. Kim, K. Lee, S.-H. Oh, G. Wang, D.-Y. Kim, G.-Y. Jung, and T. Lee, Nanotechnology 19, 405201 (2008).

${ }^{9}$ E. Linn, R. Rosezin, C. Kügeler, and R. Waser, Nat. Mater. 9, 403 (2010).

${ }^{10}$ L. Ma, S. Pyo, J. Ouyang, Q. Xu, and Y. Yang, Appl. Phys. Lett. 82, 1419 (2003).

${ }^{11}$ L. D. Bozano, B. W. Kean, V. R. Deline, J. R. Salem, and J. C. Scott, Appl. Phys. Lett. 84, 607 (2004).

${ }^{12}$ D. Tondelier, K. Lmimouni, D. Vuillaume, C. Fery, and G. Haas, Appl. Phys. Lett. 85, 5763 (2004).

${ }^{13}$ M. Cölle, M. Büchel, and D. M. de Leeuw, Org. Electron. 7, 305 (2006). 
${ }^{14}$ R. Waser, R. Dittmann, G. Staikov, and K. Szot, Adv. Mater. 21, 2632 (2009).

${ }^{15}$ B. Cho, J.-M. Yun, S. Song, Y. Ji, D.-Y. Kim, and T. Lee, Adv. Funct. Mater. 21, 3976 (2011).

${ }^{16}$ S. Nau, S. Sax, and E. J. W. List-Kratochvil, Adv. Mater. 26, 2508 (2014).

${ }^{17}$ G. Ligorio, M. V. Nardi, C. Christodoulou, I. Florea, N.-C. Monteiro, O. Ersen, M. Brinkmann, and N. Koch, Appl. Phys. Lett. 104, 163302 (2014).

${ }^{18}$ M. Kröger, S. Hamwi, J. Meyer, T. Riedl, W. Kowalsky, and A. Kahn, Appl. Phys. Lett. 95, 123301 (2009).

${ }^{19}$ K. Kanai, K. Koizumi, S. Ouchi, Y. Tsukamoto, K. Sakanoue, Y. Ouchi, and K. Seki, Org. Electron. 11, 188 (2010).

${ }^{20}$ C. H. Cheung, K. K. Tsung, K. C. Kwok, and S. K. So, Appl. Phys. Lett. 93, 083307 (2008).

${ }^{21}$ T.-Y. Chu and O.-K. Song, J. Appl. Phys. 104, 023711 (2008).
${ }^{22}$ S. L. M. van Mensfoort, V. Shabro, R. J. de Vries, R. A. J. Janssen, and R. Coehoorn, J. Appl. Phys. 107, 113710 (2010).

${ }^{23}$ See supplementary material at http://dx.doi.org/10.1063/1.4945710 for $(i)$ resistance data, (ii) description of the electric-measurements fits, and (iii) resistance-switching of bistable device.

${ }^{24}$ N. Koch, A. Kahn, J. Ghijsen, J.-J. Pireaux, J. Schwartz, R. L. Johnson, and A. Elschner, Appl. Phys. Lett. 82, 70 (2003).

${ }^{25}$ J. Niederhausen, P. Amsalem, J. Frisch, A. Wilke, A. Vollmer, R. Rieger, K. Müllen, J. P. Rabe, and N. Koch, Phys. Rev. B 84, 165302 (2011).

${ }^{26}$ H. Bässler, Phys. Status Solidi 175, 15 (1993).

${ }^{27}$ W. Pasveer, J. Cottaar, C. Tanase, R. Coehoorn, P. Bobbert, P. W. M. Blom, D. de Leeuw, and M. Michels, Phys. Rev. Lett. 94, 206601 (2005).

${ }^{28}$ D. Ielmini, R. Bruchhaus, and R. Waser, Phase Trans. 84, 570 (2011).

${ }^{29}$ P. Sebastian, F. Lindner, K. Walzer, B. Lüssem, and K. Leo, J. Appl. Phys. 110, 084508 (2011) 